\title{
THE DISTANCE TO THE HELIOSPHERIC VLF EMISSION REGION
}

\author{
R. L. McNutt, Jr,* A. J. Lazarus,** J. W. Belcher,** J. Lyon,*** \\ C. C. Goodrich $†$ and R. Kulkarni $\dagger$ \\ * The Johns Hopkins University Applied Physics Laboratory, Laurel, \\ MD 20723, U.S.A. \\ ** MIT Department of Physics and Center for Space Research, Cambridge, \\ MA 02139, U.S.A \\ *** Department of Physics and Astronomy, Dartmouth College Hanover, \\ NH 03755, U.S.A. \\ + Department of Astronomy, University of Maryland, College Park, \\ MD 20742, U.S.A.
}

\begin{abstract}
Two m?; or episodes of heliospheric VLF emissions near $3 \mathrm{kHz}$ have been observed by the Voyager spacecraft in $1983 / 84 / 1 /$ and $1992 / 3 / 2 /$. This higher-frequency component is apparently triggered by solar wind transients 13,4 / with sufficiently large spatial extents and energies to continue to propagate as shocks in the heliosheath. Entrainment of previously unshocked material and changed flow conditions in the heliosheath both tend to slow the shock propagation. The shock evolution is not self-similar. Rather, it is intermediate to two blast-wave similarity solutions in the moving solar wind frame. In one solution the shock moves as time to the $2 / 3$ power $/ 5 /$ and in the other as time to the $4 / 5$ power. Using these models, the shock/Forbush decrease observed at Voyager 2 in September, 1991 and the turn-on of the 1992 emission is consistent with an emission region distance of $-130 \mathrm{AU}$ (assuming no additional slowing of the shock in the heliosheath). If the termination shock was at $\sim 70 \mathrm{AU}$ when the transient shock collided with it, the true distance to the source region was probably closer to $\sim 115 \mathrm{AU}$.
\end{abstract}

\section{INTRODUCTION AND CURRENT STATUS OF EMISSIONS}

In 1984 low frequency $(-3 \mathrm{kHz})$ radio emissions in the outer heliosphere were detected by the Plasma Wave experiment (PWS) on both Voyager spacecraft. The strong emissions were "on" for -200 days in late 1983 and early 1984 and interpreted as coming from the vicinity of the termination shock of the solar wind /1/. Gurnett et al. /2/ reported a new high-intensity event observed in mid-1992 through early 1993. Detected by both Voyager spacecraft, these emissions are similar in spectral and temporal structure to those detected in 1983/4 but are the most intense emissions detected to date. McNutt hypothesized that the high speed streams observed in the solar wind with the Plasma Science (PLS) experiment on Voyager 2 in 1982/3 were responsible for triggering the 1983/4 emission and derived bounds of -70 AU to -140 AU to the emission region $13,4 /$. This "trigger hypothesis" was predictive: "Given the passage of such a stream by Voyager 2 , we suggest that a strong component of radio noise will again be registered by the Plasma Wave experiments on the two Voyagers in less than a year from the passage." $/ 3$ / 


\section{SOLAR ACTIVITY AND THE 1992/3 EMISSIONS}

A possibility for testing the trigger hypothesis occurred on 26 May 1991. At 35 AU, the Voyager 2 PLS experiment detected an anomalously strong shock, apparently associated with the increased level of solar activity in March 1991. McNutt et al. /6/ predicted a new strong episode of radio emission commencing near the end of 1991. By early 1992, there had been no new onset; however, a later shock did result in a renewal of the emissions $/ 2$. By applying the trigger hypothesis, Gurnett et al. $/ 2 /$ identified the shock as the the one responsible for the large Forbush decrease that passed the Earth on day 147 of 1991 and Voyager 2 about day $250 \mathrm{/7} /$ and derived a distance to the heliopause of 116 to $177 \mathrm{AU}$.

\section{THE TRIGGER EQUATION AND THE IDENTIFICATION OF THE TRIGGER}

For a disturbance propagating radially from the sun at a speed $v_{\text {diss }}$, the time delay $\Delta t$ between the time of observation at the spacecraft (located at a heliocentric distance of $r_{o b s}$ ) and the time of the observation of the the VLF radio emission can be easily calculated. Neglecting small corrections due to light transit time and spacecraft motion

$$
\Delta t \equiv t_{\text {obs }}-t_{\text {trigger }}=\int_{r_{\text {obs }}}^{r_{\text {souncet }}} \frac{d r}{v_{\text {dist }}}
$$

Uncertainty in a good quantitative estimate of the heliocentric distance to the emission site $r_{\text {source }}$ is driven by lack of knowledge of $v_{\text {dist }}$ as a function of $r$. Estimates of $r_{\text {source }}$ to date $12,3,4 /$ have been based upon constant values of $v_{\text {diss }}$.

Unique association of events on the sun with shock waves seen by interplanetary spacecraft has remained problematic. Gurnett et al. have argued that the triggers are associated with the Forbush decreases seen at Earth orbit for both the 1983/4 and 1992/3 VLF events /2/. Solar wind data from the PLS experiment on Voyager 2 shows the shock associated with the cosmic ray flux decrease on day 251 of 1991 traveling at $548 \pm 10 \mathrm{~km} \mathrm{~s}^{-1}$; the spacecraft heliocentric distance was $35.4 \mathrm{AU}$. The major Forbush decrease of 1982 on day 195 suggested for the 1982/3 trigger $/ 2 /$ passed Voyager 2 some 6 months prior to the trigger suggested originally $13,4 \%$. However, the fast streams identified by McNutt were also responsible for keeping the cosmic ray flux depressed /8/ and are associated with a second large increase in solar activity during that year. Dynamical evolution of several decrease-producing shocks driven by the high speed streams $/ 3,4 /$ is likely to have produced the "correct" major high-speed trigger structure $/ 9 /$.

\section{BLAST WAVES AND SIMILARITY SOLUTIONS}

Propagation of anomalously strong transient shocks in the interplanetary medium can be approximated by spherically symmetric blast waves. So-called similarity solutions exist if only two dimensional quantities (as well as other dimensionless parameters) are required to specify the problem $/ 10 \%$. Hydrodynamic quantities behind the shock are then only functions of a single similarity parameter $\eta \equiv r t^{-\alpha}$. For a medium with density varying as a power of $r$, these solutions do not exist for a constant speed outflow such as in the solar wind case. However, suppose we make a "local" Galilean transformation to the rest frame of the solar wind. Let primed quantities refer to the solar wind frame (taken to have a constant radial velocity $v)$. The undisturbed density varies as $\rho^{\prime}\left(r^{\prime}\right)=\rho_{0} r_{0}^{2} /\left(r^{\prime}+v t\right)^{2}$, and the blast wave occurring at $r=0$ and $t=t^{\prime}=0$ convects outward with the solar wind. The shock front moves out more rapidly than the undisturbed solar wind and is at a location $r_{\text {shock }}{ }^{\prime}=r_{\text {shock }}-v t$. Hence, there exist two limits to the undisturbed density in the primed frame of reference:

$$
\begin{array}{llll}
\text { (I) } r^{\prime} \gg v t & \rho^{\prime}\left(r^{\prime}\right) \approx r^{\prime-2} \rightarrow \alpha=\frac{2}{3} & \text { (II) } r^{\prime} \ll v t & \rho^{\prime}\left(r^{\prime}\right) \approx t^{-2} \rightarrow \alpha=\frac{4}{5}
\end{array}
$$


These two cases correspond to similarity solutions in the solar wind frame (specified by the blast wave energy $E=$ const, $\rho_{0}$, and the specific heat ratio $\gamma$ ): the first to a shock speed so much in excess of the solar wind speed that the latter is irrelevant $/ 11 /$ and the latter to a density that drops everywhere in time because we are viewing the surrounding medium from the moving frame. The true solution should have $\alpha=\alpha(r, t)$ varying from $2 / 3$ to $4 / 5$ in the vicinity of the shock front of the blast wave. Let $r=0, t=0$ correspond to the initial blast wave (day 145 of 1991) and observation of the shock by Voyager 2 to $r=35.4 \mathrm{AU}$ at $t=0.2904 \mathrm{yr}$ (day 251 of 1991). Solving for the undisturbed solar wind speed we obtain 487 and $427 \mathrm{~km} \mathrm{~s}^{-1}$ for $\alpha=2 / 3$ and $4 / 5$, respectively ( $\alpha=1$ corresponds to constant propagation at 548 $\mathrm{km} \mathrm{s}^{-1}$ and overconstrains the problem, i.e. a constant value of $v_{\text {dist }}$ is not consistent with the observations). The corresponding locations of the shock on day 188 of $1992(t=1.1178 \mathrm{yr})$ when the radio noise turned on are 128.5 and $127.8 \mathrm{AU}$, respectively. Simple propagation of the shock at a constant speed from the Voyager 2 observation gives a location of $131.0 \mathrm{AU}$.

\section{TRANSIENT PROPAGATION IN THE HELIOSHEATH}

If the region of the heliopause is the source of the emissions $/ 2 /$, then two complicating factors arise: (1) there is a change in propagation speed of the triggering disturbance at the termination shock and (2) the location of this change (the termination shock) is not known. Using the Rankine-Hugoniot conditions and ratio of specific heats $\gamma=5 / 3$, the transmitted disturbance speed is $c_{s}+v_{\text {sheath }}=0.809 V_{\text {solar wind }}$ for weak disturbances. As the strength of the transient shock is increased, the transmitted transient speed increases toward that of the ambient unshocked solar wind speed $/ 12 \%$. Then for nearly constant propagation speed through the heliosheath, the true source distance is $r_{\text {source }}=0.809 r_{\text {source }}{ }^{\prime}+0.191 r_{\text {shock }}$. Here $r_{\text {source }}$ is the source distance inferred from timing with no correction and $r_{\text {shock }}$ is the distance to the termination shock. For an inferred source distance of $-128 \mathrm{AU}$ and a shock distance of $62 \mathrm{AU} / 13 /$, the inferred true distance to the source is $\sim 115 \mathrm{AU}$. Larger distances to the shock give a limiting distance of $128 \mathrm{AU}$ (the inferred distance), close to the value expected for static pressure balance by the ram pressure alone of the interstellar medium $3,4 /$. For these cases, the Voyager 1 and 2 spacecraft may reach interstellar space during their operational lifetimes.

\section{NUMERICAL MODEL}

We have applied a full time-dependent MHD simulation code to the problem of transient propagation through the heliosphere. The simulation grid covers the region from $300 \mathrm{AU}$ upwind to $600 \mathrm{AU}$ downwind; the inner boundary is taken at $40 \mathrm{AU}$. We assume a constant speed $\left(400 \mathrm{~km} \mathrm{~s}^{-1}\right)$ solar wind with a density of $5 \mathrm{~cm}^{-3}$ at $1 \mathrm{AU}$. For interstellar conditions, we take a density of $0.05 \mathrm{~cm}^{-3}$, flow speed of $26 \mathrm{~km} \mathrm{~s}^{-1}$ and a temperature of $10^{4} \mathrm{~K}$. An interstellar magnetic field with equal components of $1 \mu \mathrm{G}$ along each axis is assumed. The code was run to 200 years at which time the upwind region is close to a steady state. The simulation gives a relatively large heliosphere with the termination shock near $-120 \mathrm{AU}$ and heliopause along the stagnation line near $\sim 160 \mathrm{AU}$.

We inject a solar wind transient and follow its evolution. We assume the transient propagates along the stagnation streamline and fills a conical region with a half-angle of $45^{\circ}$. To characterize the transient, we increase the density by a factor of 2 and step the velocity to $700 \mathrm{~km} \mathrm{~s}^{-1}$ for $7 \times 10^{5} \mathrm{~s}$. The velocity and density are then linearly ramped down over $10^{5} \mathrm{~s}$. Figure 1 shows a contour plot of the time evolution of the density. Features moving outward at a constant speed give rise to features that slope linearly upward to the right in this kind of plot. The vertical clustering of contour lines on the right hand side of the plot indicates the heliopause; a similar clustering somewhat to the left is the termination shock, where the density increases by a factor of 4 . The transient disturbance can be seen as the S-shaped jogs in the contour lines that propagate outward to the termination shock. Preliminary analysis suggests good agreement with $\alpha=2 / 3$ in the solar wind frame. Further work is required to produce a simulation quantitatively consistent with solar wind $/ 14 /$ and VLF $/ 1,2 /$ observations and known properties of the Very Local Interstellar Medium (VL:SM) /15/. 


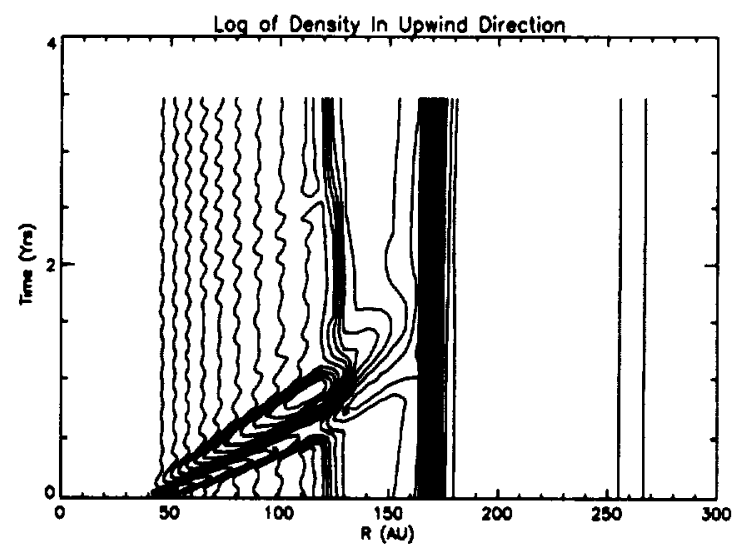

Fig. 1. Density contours (as a function of space and time) for a solar wind transient propagating in the global simulation ( $x$, horizontal, is the distance from the sun in the upwind direction for the interstellar medium; $t$, vertical, is the time in years from the injection at the inner boundary at $40 \mathrm{AU}$ ).

\section{ACKNOWLEDGEMENTS}

This work has been supported by NASA at JHU/APL under subcontract SC-A-38368 from MIT, under Task I of Navy Contract N00039-91-C-001, and at MIT under NASA contract 959203 from JPL to MIT, and under NASA grant NAGW-1550.

\section{REFERENCES}

1. W. S. Kurth, D. A. Gurnett, F. L. Scarf, and R. L. Poynter, Nature, $\underline{312}$, 27 (1984).

2. D. A. Gurnett, W. S. Kurth, S. C. Allendorf, and R. L. Poynter, Science, 262, 199 (1993).

3. R. L. McNutt, Jr., Geophys. Res. Lett., 15 , 1307 (1988).

4. R. L. McNutt, Jr., Adv.Sp.Res., 9, \#4, 235 (1989).

5. D. F. Smart and M. A. Shea, J. Geophys. Res., 90 , 183 (1985).

6. R. L. McNutt, R. L., Jr., S. Grzedzielski, A. J. Lazarus, and J. W. Belcher, EOS Trans. Am. Geophys. U., Suppl., 388, 29 Oct., 1991.

7. W. R. Webber, W. R. and J. A. Lockwood, J. Geophys. Res., 98 , 7821 (1993).

8. L. F. Burlaga, F. B. McDonald, M. L. Goldstein, and A., J. Lazarus, J. Geophys. Res., 90 , 12,027 (1985).

9. S. Grzedzielski, and A. J. Lazarus, J. Geophys. Res., 98, 5551 (1993).

10. L. I. Sedov, Similarity and Dimensional Methods in Mechanics, Academic Press, New York, 1959.

11. E. N. Parker, Interplanetary Dynamical Processes, John Wiley, New York, 1963.

12. K. Naidu, and A. Barnes, J. Geophys. Res., 99 , 11,553 (1994).

13. A. C. Cummings, E. C. Stone, and W. R. Webber, J. Geophys. Res., 99 , 11,547 (1994).

14. J. W. Belcher, A. J. Lazarus, R. L. McNutt, Jr., and G. S. Gordon, Jr., J. Geophys. Res., 98, 15,177 (1993).

15. S. T. Suess, Rev. Geophys., 28 , 97 (1990). 